\title{
Multiplicity of solutions for Robin problem involving the $p(x)$-laplacian
}

\author{
Hassan Belaouidel \\ Faculty of Sciences, University \\ Mohamed I, Oujda, Morocco \\ email: belaouidelhassan@hotmail.fr
}

\author{
Anass Ourraoui \\ Department of Mathematics and \\ Computer Science, Faculty of Sciences, \\ University Mohamed I, Oujda, Morocco \\ email: anas.our@hotmail.com
}

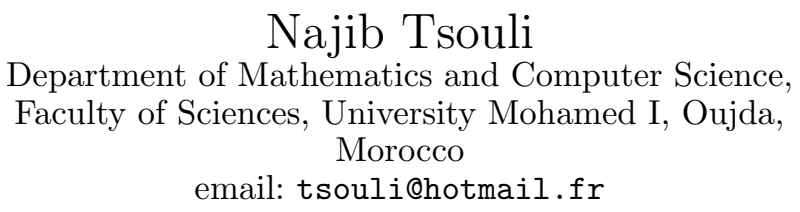

email: tsouli@hotmail.fr

Abstract. This paper is concerned with the existence and multiplicity of solutions for $p(x)$-Laplacian equations with Robin boundary condition. Our technical approach is based on variational methods.

\section{Introduction}

The purpose of this paper is to study the existence and multiplicity of solutions for the following Robin problem involving the $p(x)$-Laplacian

$$
\left\{\begin{array}{lll}
-\operatorname{div}\left(|\nabla u|^{p(x)-2} \nabla u\right)=f(x, u) & \text { in } & \Omega \\
|\nabla u|^{p(x)-2} \frac{\partial u}{\partial v}=\beta(x)|u|^{p(x)-2} u & \text { on } & \partial \Omega
\end{array}\right.
$$

2010 Mathematics Subject Classification: 39A05, 34B15

Key words and phrases: $p(x)$-Laplacian, mountain pass theorem, multiple solutions, critical point theory 
where $\Omega$ is an open bounded subset of $\mathbb{R}^{N}(N \geq 2)$, with smooth boundary, $\frac{\partial u}{\partial v}$ is the outer unit normal derivative on $\partial \Omega, \beta \in \mathrm{L}^{\infty}(\Omega)$, with $\operatorname{ess} \inf _{\Omega} \beta>0$ and $p \in C_{+}(\bar{\Omega})$ with

$$
1<p^{-}:=\inf _{\bar{\Omega}} p(x) \leq p^{+}:=\sup _{\bar{\Omega}} p(x)<+\infty .
$$

Recently, there has been an increasing interest in studying of problems (1). This great interest may be justified by their various physical applications, we can for example refer to $[3,2,6,9,16,19,23,24,25,27,30,32]$. In fact, there are applications concerning elastic mechanics [33], electrorheological fluids [28, 29], image restoration [12], dielectric breakdown, electrical resistivity and polycrystal plasticity and continuum mechanics [4]. We refer to [18] for an overview of this subject and to $[11,14]$ for the $p(x)$-Laplacian equations.

From the variational point of view, by using a theorem obtained by B. Ricceri in [5], the work [2] shows the existence of at least three solutions for a Navier problem involving the fourth order operator.

The authors in [6] obtained the existence of three distinct weak solutions of $p(x)$-Laplacian Dirichlet problems as applications of critical point theorem obtained by G. Bonanno and S.A. Marano in [7]. In the same breath, the authors in [30] consider the $p(x)$-Laplacian-like problem ( originated from a capillary phenomenon) which the main tool is a general critical point theorem in $[8]$.

In the statement of problem (1), $f: \Omega \times \mathbb{R} \rightarrow \mathbb{R}$ is a Carathéodory function verifying $\left(F_{0}\right)$ such that

$\left(F_{0}\right)$ There exists a constant $c_{1} \geq 0$ such that

$$
|f(x, t)| \leq c_{1}\left(1+|t|^{q(x)-1}\right),
$$

for all $(x, t) \in \Omega \times \mathbb{R}$ where $\mathrm{q} \in \mathrm{C}_{+}(\bar{\Omega}), \mathrm{q}(\mathrm{x})<\mathrm{p}^{*}(\mathrm{x})$ for all $x \in \bar{\Omega}$.

Where

$$
p^{*}(x)=\left\{\begin{array}{lll}
\frac{N p(x)}{N-p(x)} & \text { if } & p(x)<N \\
+\infty & \text { if } & p(x) \geq N
\end{array}\right.
$$

Motivated by the references mentioned above, we establish the existence and multiplicity of solutions for problem (1). It is known that the extension $p(x)$ Laplace operator possesses more complicated structure than the p-Laplacian. For example, it is inhomogeneous and usually it does not have the so-called 
first eigenvalue, since the infimum of its spectrum is zero. This provokes some mathematical difficulties which makes the study of such a problems particulary interesting.

Now, we formulate our main results as follows.

Theorem 1 Assume that $\left(\mathrm{F}_{0}\right)$ and the following assumptions hold.

$\left(F_{1}\right) 0<\lim _{t \rightarrow 0} \frac{F(x, t)}{\left.|t|\right|^{-}}<\frac{1}{p^{-}}$, for $|t|>\delta$, with $\delta>0$,

$\left(F_{2}\right) \lim _{|t| \rightarrow+\infty} \frac{p(x) F(x, t)}{|t|^{-}} \leq 0$ a.e $x \in \Omega$,

$\left(F_{3}\right) \lim _{|t| \rightarrow+\infty} \int_{\Omega} F(x, t) d x=-\infty$,

Then the problem (1) has two weak solutions.

Theorem 2 Assume that $\left(\mathrm{F}_{0}\right)$ and the following conditions hold.

$\left(F_{4}\right) \lim _{|t| \rightarrow+\infty} \frac{F(x, t)}{\left.|t|\right|^{-}}=0$,

$\left(F_{5}\right) f(x, t) t>0$ for all $(x, t) \in \Omega \times \mathbb{R}$,

$\left(F_{6}\right) \lim _{|t| \rightarrow+\infty}\left[f(x, t) t-p^{+} F(x, t)\right]=-\infty$.

Then the problem (1) has at least one weak solution.

Through taking the same methods of this paper, results similar to Theorems 1-2 can also be proven for Neumann and Steklov problems.

Our paper is organized as follows. We first present some necessary preliminary results on variable exponent Sobolev spaces. Next, we give the proof of the main results about the existence of weak solutions.

\section{Preliminaries}

In the sequel, let $p(x) \in C_{+}(\bar{\Omega})$, where

$$
C_{+}(\bar{\Omega})=\{h: h \in C(\bar{\Omega}), h(x)>1 \text { for any } x \in \bar{\Omega}\} .
$$

The variable exponent Lebesgue space is defined by

$$
\mathrm{L}^{\mathrm{p}(\mathrm{x})}(\Omega)=\left\{\mathrm{u}: \Omega \rightarrow \mathbb{R} \text { measurable and } \int_{\Omega}|\mathrm{u}(\mathrm{x})|^{\mathrm{p}(\mathrm{x})} \mathrm{d} \mathrm{x}<+\infty\right\}
$$


furnished with the Luxemburg norm

$$
|u|_{L^{p(x)}(\Omega)}=|u|_{p(x)}=\inf \left\{\sigma>0: \int_{\Omega}\left|\frac{u(x)}{\sigma}\right|^{p(x)} d x \leq 1\right\},
$$

and the variable exponent Sobolev space is defined by

$$
\mathrm{W}^{1, p(x)}(\Omega)=\left\{u \in \mathrm{L}^{\mathrm{p}(\mathrm{x})}(\Omega):|\nabla u| \in \mathrm{L}^{\mathrm{p}(\mathrm{x})}(\Omega)\right\}
$$

equipped with the norm

$$
\|u\|_{W^{1, p(x)}(\Omega)}=|u|_{L^{p(x)}(\Omega)}+|\nabla u|_{L^{p(x)}(\Omega)} .
$$

Proposition 1 [21] The spaces $\mathrm{L}^{\mathrm{p}(\mathrm{x})}(\Omega)$ and $\mathrm{W}^{1, \mathrm{p}(\mathrm{x})}(\Omega)$ are separable, uniformly convex, reflexive Banach spaces. The conjugate space of $\mathrm{L}^{\mathrm{p}(\mathrm{x})}(\Omega)$ is $\mathrm{L}^{\mathrm{q}(\mathrm{x})}(\Omega)$, where $\mathrm{q}(\mathrm{x})$ is the conjugate function of $\mathrm{p}(\mathrm{x})$; i.e.,

$$
\frac{1}{p(x)}+\frac{1}{q(x)}=1
$$

for all $\mathrm{x} \in \Omega$. For $\mathrm{u} \in \mathrm{L}^{\mathrm{p}(\mathrm{x})}(\Omega)$ and $v \in \mathrm{L}^{\mathrm{q}(\mathrm{x})}(\Omega)$ we have

$$
\left|\int_{\Omega} u(x) v(x) d x\right| \leq\left(\frac{1}{p^{-}}+\frac{1}{q^{-}}\right)|u|_{p(x)}|v|_{q(x)} .
$$

Proposition 2 [21] For $\mathrm{p}, \mathrm{r} \in \mathrm{C}_{+}(\bar{\Omega})$ such that $\mathrm{r}(\mathrm{x}) \leq \mathrm{p}^{*}(\mathrm{x})\left(\mathrm{r}(\mathrm{x})<\mathrm{p}^{*}(\mathrm{x})\right)$ for all $\mathrm{x} \in \bar{\Omega}$, there is a continuous (compact) embedding

$$
W^{1, p(x)}(\Omega) \hookrightarrow \mathrm{L}^{\mathrm{r}(\mathrm{x})}(\Omega),
$$

where

$$
p^{*}(x)=\left\{\begin{array}{lll}
\frac{N p(x)}{N-p(x)} & \text { if } p(x)<N \\
+\infty & \text { if } p(x) \geq N .
\end{array}\right.
$$

Proposition 3 [15] For $\mathrm{p} \in \mathrm{C}_{+}(\bar{\Omega})$ and such $\mathrm{r} \in \mathrm{C}_{+}(\partial \Omega)$ that $\mathrm{r}(\mathrm{x}) \leq \mathrm{p}^{\partial}(\mathrm{x})$ $\left(\mathrm{r}(\mathrm{x})<\mathrm{p}^{\partial}(\mathrm{x})\right)$ for all $\mathrm{x} \in \bar{\Omega}$, there is a continuous (compact) embedding

$$
W^{1, p(x)}(\Omega) \hookrightarrow L^{r(x)}(\partial \Omega),
$$

where

$$
p^{\partial}(x)=(p(x))^{\partial}:=\left\{\begin{array}{lll}
\frac{(N-1) p(x)}{N-p(x)} & \text { if } p(x)<N \\
+\infty & \text { if } p(x) \geq N
\end{array}\right.
$$


Proposition 4 [17], [Theorem 2.1] For any $\mathfrak{u} \in \mathrm{W}^{1, p(x)}(\Omega)$, let

$$
\|\mathfrak{u}\|_{\partial}:=|u|_{L^{p(x)}(\partial \Omega)}+|\nabla u|_{L^{p(x)}(\Omega)} .
$$

Then $\|\mathrm{u}\|_{\partial}$ is a norm on $\mathrm{W}^{1, p(x)}(\Omega)$ which is equivalent to

$$
\|u\|_{W^{1}, p(x)(\Omega)}=|u|_{L^{p(x)}(\Omega)}+|\nabla u|_{L^{p(x)}(\Omega)} .
$$

Now, for any $u \in X:=W^{1, p(x)}(\Omega)$ define

$$
\|u\|:=\inf \left\{\sigma>0: \int_{\Omega}\left(\left|\frac{\nabla u(x)}{\sigma}\right|^{p(x)} d x+\int_{\partial \Omega} \beta(x)\left|\frac{u(x)}{\sigma}\right|^{p(x)}\right) d \sigma_{x} \leq 1\right\} .
$$

Where $\beta \in \mathrm{L}^{\infty}(\Omega)$ and $d \sigma_{x}$ is the measure on the boundary $\partial \Omega$. Then by (4), $\|\cdot\|$ is also a norm on $W^{1, p(x)}(\Omega)$ which is equivalent to $\|\cdot\|_{W^{1, p(x)}(\Omega)}$ and $\|\cdot\|_{\partial}$. Now, we introduce the modular $\rho: X \rightarrow \mathbb{R}$ defined by

$$
\rho(u)=\int_{\Omega}|\nabla u|^{p(x)} \mathrm{d} x+\int_{\partial \Omega} \beta(x)|u(x)|^{p(x)} \mathrm{d} \sigma_{x}
$$

for all $u \in X$. Here, we give some relations between the norm $\|$.$\| and the$ modular $\rho$.

Proposition 5 [21] For $\mathrm{u} \in \mathrm{X}$ we have

(i) $\|u\|<1(=1 ;>1) \Leftrightarrow \rho(u)<1(=1 ;>1)$;

(ii) If $\|\mathrm{u}\|<1 \Rightarrow\|\mathrm{u}\|^{\mathrm{p}^{+}} \leq \rho(\mathrm{u}) \leq\|\mathrm{u}\|^{\mathrm{p}^{-}}$;

(iii) If $\|u\|>1 \Rightarrow\|u\|^{p^{-}} \leq \rho(u) \leq\|u\| \|^{p^{+}}$.

Lemma 1 [26] Let $\mathrm{X}=\mathrm{X}_{1} \oplus \mathrm{X}_{2}$, where $\mathrm{X}$ is a real Banach space and $\mathrm{X}_{2} \neq 0$, and is finite dimensional. Suppose that $\phi \in \mathrm{C}^{1}(\mathrm{X}, \mathrm{R})$ satisfies Cerami condition (C) with the following assertions:

(i) There is a constant $\alpha$ and a bounded neighborhood $\mathrm{D}$ of 0 in $\mathrm{X}_{2}$ such that $\phi \mid \partial \mathrm{D} \leq \alpha$.

(ii) There is a constant $\beta>\alpha$ such that $\phi \mid X_{1} \geq \beta$.

Then $\phi$ possesses a critical value $\mathbf{c}$, moreover, $\mathrm{c}$ can be characterized as

$$
c=\inf _{h \in \Gamma} \max _{u \in \bar{D}} \phi(h(u))
$$

where

$$
\Gamma=\{h \in C(D, X) \mid h=i d \text { on } \partial D\} .
$$


Lemma 2 [10] Let $\mathrm{X}=\mathrm{X}_{1} \oplus \mathrm{X}_{2}$, where $\mathrm{X}$ is a real Banach space and $\mathrm{X}_{2} \neq 0$, and is finite dimensional. Suppose that $\phi \in \mathrm{C}^{1}(\mathrm{X}, \mathrm{R})$ satisfies Palais-smalle condition $(P S)$ with the following assertions for some $r>0$ :

(i) $\phi(u) \leq 0$, for $u \in X_{1},\|u\| \leq r$.

(ii) $\phi(u) \geq 0$, for $u \in X_{2},\|u\| \leq r$.

Assume also that $\phi$ is bounded below and $\inf _{\mathrm{X}} \phi<0$. Then $\phi$ has at least two nonzero critical points.

Definition 1 We say that $\mathfrak{u} \in \mathrm{X}$ is a weak solution of (1) if

$$
\int_{\Omega}|\nabla u|^{p(x)-2} \nabla u \nabla v d x+\int_{\partial \Omega} \beta(x)|u|^{p(x)-2} u v d \sigma_{x}=\int_{\Omega} f(x, u) v d x
$$

for all $v \in X$.

The functional associated to (1) is given by

$$
\phi(u)=\int_{\Omega} \frac{1}{p(x)}|\nabla u|^{p(x)} d x+\int_{\partial \Omega} \frac{1}{p(x)} \beta(x)|u|^{p(x)} d x-\int_{\Omega} F(x, u) d x
$$

It should be noticed that under the condition $\left(F_{0}\right)$ the functional $\phi$ is of class $C^{1}(X, \mathbb{R})$ and

$$
\begin{array}{r}
\phi^{\prime}(\mathrm{u}) \cdot v=\int_{\Omega}|\nabla u|^{p(x)-2} \nabla u \nabla v \mathrm{~d} x+\int_{\partial \Omega} \beta(x)|u|^{p(x)-2} u v d \sigma_{x}-\int_{\Omega} f(x, u) v d x \\
\forall u, v \in X .
\end{array}
$$

Then, we can see that the weak solution of (1) corresponds to critical point of the functional $\phi$.

\section{Proof of main result}

We recall that $\phi$ satisfies Palais-smale condition (PS) in $X$, if any sequence $\left(u_{n}\right)$ such that $\phi\left(u_{n}\right)$ is bounded and $\phi^{\prime}\left(u_{n}\right) \rightarrow 0$ as $n \rightarrow+\infty$, has convergent subsequence.

\section{Proof of Theorem 1}

Let start by the following lemma. 
Lemma 3 Any bounded sequence (PS) of $\phi$ has a strongly convergent subsequence.

Proof. Let $\left(u_{n}\right) \subset X$ be a sequence bounded (PS) sequence of $\phi$. Up to a subsequence, we may find $u \in X$ such that $u_{n} \rightarrow u$.

From the growth condition $\left(F_{0}\right)$ and Sobolev embedding, we have that $\int_{\Omega} f(x, u)\left(u_{n}-u\right) d x \rightarrow 0$, since $\phi^{\prime}\left(u_{n}\right)\left(u_{n}-u\right) \rightarrow 0$ then

$$
\int_{\Omega}\left|\nabla u_{n}\right|^{p(x)-2} \nabla u \nabla\left(\left(u_{n}-u\right)\right) d x+\int_{\partial \Omega} \beta(x)\left|u_{n}\right|^{p(x)-2} u_{n}\left(u_{n}-u\right) d \sigma_{x} \rightarrow 0 .
$$

As the mapping $A: W^{1, p(x)}(\Omega) \rightarrow \mathbb{R}$ defined by

$$
\langle A u, v\rangle=\int_{\Omega}|\nabla u|^{p(x)-2} \nabla u \nabla v d x+\int_{\partial \Omega} \beta(x)|u|^{p(x)-2} u v d \sigma_{x}
$$

for all $u, v \in X$ is of type $\left(S_{+}\right)$, so $u_{n} \rightarrow u$ in $W^{1, p(x)}(\Omega)$.

Lemma 4 The functional $\phi$ is coercive, that is, $\phi(u) \rightarrow+\infty$ when $\|\mathrm{u}\| \rightarrow$ $+\infty$.

Proof. Suppose that there exist $\left(u_{n}\right) \subset X$ and a positive constant $C$ such that

$$
\left\|u_{n}\right\| \rightarrow+\infty, \quad \phi\left(u_{n}\right) \leq C .
$$

Putting $v_{n}=\frac{\mathfrak{u}_{n}}{\left\|u_{n}\right\|}$, so we may find $v \in X$ and a subsequence of $\left(v_{n}\right)$ still denoted by $\left(v_{n}\right)$ such that $v_{n} \rightarrow v$ in $X$ and $v_{n} \rightarrow v$ in $L^{p(x)}(\Omega)$.

By $\left(F_{1}\right)$, for any $\epsilon>0, \exists L>0$ such that

$$
F(x, t) \leq \frac{\varepsilon}{p(x)}|t|^{p^{-}} \forall|t|>\text { L a.e } x \in \Omega,
$$

thus, we may find a positive constant $C$ such that

$$
F(x, t) \leq \frac{\varepsilon}{p(x)}|t|^{p^{-}}+C \forall t \in \mathbb{R} \text { a.e } x \in \Omega .
$$

Therefore,

$$
\frac{C}{\left\|u_{n}\right\|^{p^{-}}} \geq \frac{\phi\left(u_{n}\right)}{\left\|u_{n}\right\|^{p^{-}}} \geq \frac{1}{p^{+}} \frac{1}{\left\|u_{n}\right\|^{p^{-}}}\left[\int_{\Omega}\left|\nabla u_{n}\right|^{p(x)} d x+\int_{\partial \Omega} \beta(x)\left|u_{n}\right|^{p(x)} d \sigma_{x}\right]
$$




$$
\begin{gathered}
-\frac{\epsilon}{p^{+}} \int_{\Omega}\left|v_{n}\right|^{p^{-}} \mathrm{d} x-\frac{\mathrm{C}|\Omega|}{\left\|\mathrm{u}_{\mathrm{n}}\right\| \mathrm{p}^{-}} \\
\geq \frac{1}{\mathrm{p}^{+}}-\frac{\epsilon}{\mathrm{p}^{+}} \int_{\Omega}\left|v_{\mathrm{n}}\right|^{\left.\right|^{-}} \mathrm{d} x-\frac{\mathrm{C}|\Omega|}{\left\|\mathrm{u}_{\mathrm{n}}\right\|^{\mathrm{p}^{-}}} .
\end{gathered}
$$

Consequently, choosing $\epsilon$, such that $\int_{\Omega}\left|v_{n}\right|^{p^{-}} d x>C_{0}$, where $C_{0}$ is the best constant in the embedding $W^{1, p(x)}(\Omega) \hookrightarrow \mathrm{L}^{\mathrm{p}^{-}}(\Omega)$.

On the other hand, because $\|v\|_{W^{1, p(x)}(\Omega)} \leq \liminf \left\|v_{n}\right\|=1$ by

$$
\int_{\Omega}|\nabla v|^{p^{-}} \mathrm{d} x+\int_{\Omega}|v|^{p^{-}} \mathrm{d} x \leq \mathrm{C}_{0}
$$

so we get $\int_{\Omega} \nabla|v|^{p^{-}} \mathrm{dx}=0$, which means that $v=$ constant $\neq 0$.

We obtain

$$
\lim _{\left|u_{n}\right| \rightarrow \infty} \int_{\Omega} F\left(x, u_{n}\right) d x \rightarrow-\infty .
$$

When $\left\|u_{n}\right\| \rightarrow+\infty,\left|u_{n}\right| \rightarrow+\infty$, thereby,

$$
\begin{aligned}
C & \geq \frac{1}{p^{+}}\left[\int_{\Omega}\left|\nabla u_{n}\right|^{p(x)} \mathrm{d} x+\int_{\partial \Omega} \beta(x)\left|u_{n}\right|^{p(x)} d \sigma_{x}\right]-\int_{\Omega} F\left(x, u_{n}\right) d x \\
& \geq \frac{\left\|u_{n}\right\|^{p^{-}}}{p^{+}}-\frac{\epsilon}{p^{+}} \int_{\Omega}\left|v_{n}\right|^{p^{-}} \mathrm{d} x-\frac{C|\Omega|}{\left\|u_{n}\right\|^{p^{-}}},
\end{aligned}
$$

which implies that $\phi$ is coercive and bounded from below.

Now verifying the conditions (i) and (ii) in Lemma 2 .

The same idea from [1] and Chung [13], we have $W^{1, p(x)}(\Omega)=W_{0} \oplus \mathbb{R}$. If $u \in \mathbb{R}$, for $\|u\|<\rho, \rho>0$ and by (2)

$$
\begin{aligned}
\phi(u) & =\int_{\Omega} \frac{1}{p(x)}|\nabla u|^{p(x)} d x+\int_{\partial \Omega} \frac{1}{p(x)} \beta(x)|u|^{p(x)} d \sigma_{x}-\int_{\Omega} F(x, u) d x \\
& =-\int_{\Omega} F(x, u) d x \\
\leq &
\end{aligned}
$$

If $u \in W_{0}=\left\{z \in W^{1, p(x)}(\Omega) / \int_{\Omega} z d x=0\right\}$, from $\left(F_{0}\right)$ and $\left(F_{1}\right)$

$$
F(x, t) \leq\left(\frac{1}{p^{-}}-\epsilon\right)|u|^{p^{-}}+C \int_{\Omega}|u|^{q(x)} d x .
$$


In virtue of the the continuous embedding $X$ into $\mathrm{L}^{\mathrm{p}^{-}}(\Omega)$ and $\mathrm{L}^{\mathrm{p}^{+}}(\Omega)$,

$$
\begin{aligned}
& \phi(u) \geq \frac{1}{p^{+}}\|u\|^{p^{+}}-\int_{\Omega} F(x, u) d x \\
& \geq\left.\frac{1}{p^{+}}\|u\|\right|^{p^{+}}-\frac{1}{p^{-}} \int_{\Omega}|u|^{p^{-}} d x+\epsilon \int_{\Omega}|u|^{p^{-}} d x-C \int_{\Omega}|u|^{q(x)} d x \\
& \geq C(\epsilon)\|u\|^{p^{+}}-C\|u\|^{q^{+}}-C\|u\|^{q^{-}} \text {, }
\end{aligned}
$$

for $\|u\|=\rho$ small enough then $\phi(u) \geq 0$ for $\|u\| \leq \rho \forall u \in W_{0}$. On the other side, when $\inf _{X} \phi(u)=0$ then $\forall u \in \mathbb{R}$ is a minimum of $\phi$, that means $\phi$ admits infinite critical points.

When $u \in X$ with $i n f_{X} \phi(u)<0$, by applying Lemma $2, \phi$ has at least two nontrivial critical points, then the problem (1) has two nontrivial solutions in $\mathrm{X}$.

Proof of Theorem 2 We recall the following important inequality (cf.[22])

Lemma 5 (Poincaré-Writingers inequality) There exists a positive constant C such that for any $\mathrm{u} \in \mathrm{W}_{0}$ we have

$$
|\mathfrak{u}|_{\mathfrak{p}(x)} \leq \underline{\mathrm{C}}|\nabla \mathfrak{u}|_{\mathfrak{p}(x)}
$$

Lemma 6 Suppose that the conditions $\left(\mathrm{F}_{0}\right),\left(\mathrm{F}_{4}\right)$ and $\left(\mathrm{F}_{6}\right)$ are hold. Then $\phi$ verifies the Cerami condition $(\mathrm{C})_{\mathrm{c}}$.

Proof. Let $K \in \mathbb{R}$ such that

$$
\left|\phi\left(u_{n}\right)\right| \leq K
$$

and

$$
\left(1+\left\|u_{n}\right\|\right) \phi^{\prime}\left(u_{n}\right) \rightarrow 0 \text { in } X^{*} .
$$

Suppose that $\left\|u_{n}\right\| \rightarrow+\infty$ as $n \rightarrow+\infty$. Taking $v_{n}=\frac{u_{n}}{\left\|u_{n}\right\|}$, so

$$
v_{n} \rightarrow v \text { in } X
$$

Thus,

$$
v_{\mathrm{n}}(x) \rightarrow v(x) \text { a.e } x \in \Omega
$$

and

$$
v_{n} \rightarrow v \text { in } \mathrm{L}^{\mathrm{p}(\mathrm{x})}(\Omega) .
$$

Let $h \in X$, according to (3) we have that, 


$$
\begin{aligned}
\left.\left|\int_{\Omega}\right| \nabla u_{n}\right|^{p(x)-2} \nabla u_{n} \nabla h d x & +\int_{\partial \Omega} \beta(x)\left|u_{n}\right|^{p(x)-2} u_{n} h d \sigma_{x} \\
& -\int_{\Omega} f\left(x, u_{n}\right) h d x \mid \leq \frac{\epsilon_{n}\|h\|}{1+\left\|u_{n}\right\|} .
\end{aligned}
$$

Dividing (4) by $\left\|u_{n}\right\|^{p^{--1}}$ we have

$$
\begin{array}{r}
\left.\frac{1}{\left.\left\|u_{n}\right\|\right|^{--1}}\left|\int_{\Omega}\right| \nabla u_{n}\right|^{p(x)-2} \nabla u_{n} \nabla h d x+\int_{\partial \Omega} \beta(x)\left|u_{n}\right|^{p(x)-2} u_{n} h d \sigma_{x} \\
-\int_{\Omega} f\left(x, u_{n}\right) h d x \mid \leq \frac{\epsilon_{n}\|h\|}{\left(\left.\left\|u_{n}\right\|\right|^{--1}\right)\left(1+\left\|u_{n}\right\|\right)} .
\end{array}
$$

Then

$$
\begin{aligned}
\left.\frac{1}{\left.\left\|u_{n}\right\|\right|^{p^{-}-1}}\left|\int_{\Omega}\right| \nabla u_{n}\right|^{p(x)-2} \nabla u_{n} \nabla h d x & +\int_{\partial \Omega} \beta(x)\left|u_{n}\right|^{p(x)-2} u_{n} h d \sigma_{x} \\
& -\int_{\Omega} f\left(x, u_{n}\right) h d x \mid \leq \frac{\epsilon_{n}\|h\|}{1+\left\|u_{n}\right\|} .
\end{aligned}
$$

Since $\left\|u_{n}\right\|^{p(x)-1} \geq\left\|u_{n}\right\|^{p^{--1}}>1$,

$$
\begin{aligned}
& \left.\frac{1}{\left.\left\|u_{n}\right\|\right|^{p^{-}-1}}\left|\int_{\Omega}\right| \nabla u_{n}\right|^{p(x)-2} \nabla u_{n} \nabla h d x \\
& \quad+\int_{\partial \Omega} \beta(x)\left|u_{n}\right|^{p(x)-2} u_{n} h d \sigma_{x}-\int_{\Omega} f\left(x, u_{n}\right) h d x \mid \\
& \geq\left.\frac{1}{\left\|u_{n}\right\|^{p^{-}-1}}\left|\int_{\Omega}\right| \nabla u_{n}\right|^{p(x)-2} \nabla u_{n} \nabla h d x+\int_{\partial \Omega} \beta(x)\left|u_{n}\right|^{p(x)-2} u_{n} h d \sigma_{x} \mid \\
& \quad-\frac{1}{\left\|u_{n}\right\|^{p^{-}-1}}\left|\int_{\Omega} f\left(x, u_{n}\right) h d x\right| \\
& \geq\left.\left|\int_{\Omega}\right| \nabla v_{n}\right|^{p(x)-2} \nabla v_{n} \nabla h d x+\int_{\partial \Omega} \beta(x)\left|v_{n}\right|^{p(x)-2} v_{n} h d \sigma_{x} \mid
\end{aligned}
$$




$$
-\frac{1}{\left\|u_{n}\right\|^{p^{-}-1}}\left|\int_{\Omega} f\left(x, u_{n}\right) h d x\right|
$$

Consequently

$$
\begin{aligned}
& \left.\left|\int_{\Omega}\right| \nabla v_{n}\right|^{p(x)-2} \nabla v_{n} \nabla h d x+\int_{\partial \Omega} \beta(x)\left|v_{n}\right|^{p(x)-2} v_{n} h d \sigma_{x} \mid \\
& \quad-\frac{1}{\left\|u_{n}\right\|^{p^{-}-1}}\left|\int_{\Omega} f\left(x, u_{n}\right) h d x\right| \leq \frac{\epsilon_{n}\|h\|}{1+\left\|u_{n}\right\|}
\end{aligned}
$$

with $\epsilon_{\mathrm{n}} \rightarrow 0$ and $\mathrm{h} \in \mathrm{X}$.

By $\left(F_{0}\right),\left(F_{4}\right)$ and $\left(F_{6}\right)$ we conclude that $\frac{f\left(x, u_{n}\right)}{\left\|u_{n}\right\|^{p^{-}-1}}$ is bounded in $\left(L^{p^{-}}(\Omega)\right)^{*}$ which is separable and reflexive space, then up to a subsequence denoted also $\left(\frac{f\left(x, u_{n}\right)}{\left\|u_{n}\right\|^{p^{-}-1}}\right)$, we have $\frac{f\left(x, u_{n}\right)}{\left\|u_{n}\right\|^{p^{-}-1}} \rightarrow \widetilde{f}$, in $\left(L^{\mathfrak{p}^{-}}(\Omega)\right)^{*}$. Since $\frac{f\left(x, u_{n}\right)}{\left\|\mathfrak{u}_{n}\right\| \|^{--1}} \rightarrow 0$ a.e $x \in \Omega$, hence

$$
\frac{f\left(x, u_{n}\right)}{\left\|u_{n}\right\|^{p^{-}-1}} \rightarrow 0 \text { in }\left(L^{p^{-}}(\Omega)\right)^{*}
$$

Therefore, taking $h=v_{n}-v \in X$, in (6)

$$
\int_{\Omega}\left|\nabla v_{n}\right|^{p(x)-2} \nabla v_{n} \nabla\left(v_{n}-v\right) \mathrm{d} x+\int_{\partial \Omega} \beta(x)\left|v_{n}\right|^{p(x)-2} v_{n}\left(v_{n}-v\right) d \sigma_{x} \rightarrow 0 .
$$

By $\left(S_{+}\right)$type of the operator

$$
\mathrm{L}(\mathrm{u}) \cdot v=\int_{\Omega}|\nabla u|^{p(x)-2} \nabla u \nabla v \mathrm{~d} x+\int_{\partial \Omega} \beta(x)|u|^{p(x)-2} u v d \sigma_{x},
$$

we have $v_{n} \rightarrow v$ in $X$, so $v \neq 0$. Since $|\phi(u)| \leq K$ we obtain

$$
p^{+} \phi(u) \geq-p^{+} K
$$

Taking $h=u_{n}$, in (4)

$$
-\int_{\Omega}\left|\nabla u_{n}\right|^{p(x)} d x+\int_{\partial \Omega} \beta(x)\left|u_{n}\right|^{p(x)} d \sigma_{x}+\int_{\Omega} f\left(x, u_{n}\right) u_{n} d x \geq-\epsilon_{n}
$$

Then

$$
-p^{-} \int_{\Omega} \frac{\left|\nabla u_{n}\right|^{p(x)}}{p(x)} d x+\int_{\partial \Omega} \beta(x) \frac{\left|u_{n}\right|^{p(x)}}{p(x)} d \sigma_{x}+\int_{\Omega} f\left(x, u_{n}\right) u_{n} d x \geq-\epsilon_{n} .
$$


Adding (7)to (8), we obtain

$$
\int_{\Omega} f\left(x, u_{n}\right) u_{n} d x-p^{+} \int_{\Omega} F\left(x, u_{n}\right) d x \geq C
$$

Obviously, this is contradiction and then the proof of Lemma 6 is reached.

Lemma 7 Suppose that the conditions $\left(\mathrm{F}_{5}\right)$ and $\left(\mathrm{F}_{6}\right)$ hold, then the function $\phi / \mathbb{R}$ is anti-coercive.

Proof. From $\left(F_{6}\right)$, for all $K>0$ there exists $R>0$ such that $p^{+} F(x, u) \geq$ $f(x, u) u \geq K$ for a.e $x \in \Omega, u \in \mathbb{R}$ and thus for all $u \in \mathbb{R}$,

$$
\int_{\Omega} \mathrm{F}(x, \mathrm{u}) \mathrm{d} x \geq \frac{1}{\mathrm{p}^{+}} \mathrm{K}|\Omega|-\mathrm{c}|\Omega|,
$$

hence

$$
\int_{\Omega} \mathrm{F}(\mathrm{x}, \mathrm{u}) \mathrm{d} x \rightarrow+\infty \text { when }|\mathrm{u}| \rightarrow+\infty .
$$

By (2) and $\mathrm{K}$ is arbitrary

$$
\phi(u)=\int_{\partial \Omega} \beta(x) \frac{|u|^{p(x)}}{p(x)} d \sigma_{x}-\int_{\Omega} F(x, u) d x \geq-\int_{\Omega} F(x, u) d x
$$

Then

$$
\phi(u) \rightarrow-\infty \text { when }|u| \rightarrow+\infty \text {. }
$$

Lemma 8 Under the hypothesis $\left(\mathrm{F}_{4}\right)$, we have $\inf _{W_{0}} \phi>-\infty$.

Proof. Let $u \in W_{0}$ with $\|u\|>1$ By $\left(F_{5}\right)$, for $\epsilon>0$, we may find $K(\epsilon)>0$ such that $\mathrm{F}(x, u) \leq \epsilon|u|^{p^{-}}+K(\epsilon)$, for a.e $x \in \Omega$ and for all $u \in \mathbb{R}$.Hence,

$$
\begin{aligned}
\mathrm{F}(x, \mathrm{u}) & \leq \epsilon \int_{\Omega}|\mathrm{u}|^{p^{-}}+\mathrm{K}(\epsilon)|\Omega| \\
& \leq \epsilon \mathrm{C}\|\mathrm{u}\|^{p^{-}}+\mathrm{K}(\epsilon)|\Omega|
\end{aligned}
$$

Then, when $u \in W_{0}$ we have

$$
\begin{aligned}
\phi(u) & =\int_{\Omega} \frac{1}{p(x)}|\nabla u|^{p(x)}+\int_{\partial \Omega} \frac{1}{p(x)} \beta(x)|u|^{p(x)} d \sigma_{x}-\int_{\Omega} F(x, u) d x \\
& \geq \bar{C}\|u\|^{p^{+}}-\epsilon C\|u\|^{p^{-}}-K(\epsilon)|\Omega| \\
& \geq-K(\epsilon)|\Omega| .
\end{aligned}
$$


It follows that $\inf _{W_{0}} \phi>-\infty$.

According to previous Lemmas 6, 7 and 8, the assumptions of Lemma 1 are satisfied and then the proof of Theorem 2 is achieved.

\section{Acknowledgements}

We would like to thank the referee for carefully reading our manuscript and for giving helpful comments.

\section{References}

[1] M. Allaoui, A. El Amrouss and A. Ourraoui, On a Class of Nonlocal p(x)Laplacian Neumann Problems, Thai Journal of Mathematics, Volume 15 (2017) Number 1, 91-105.

[2] A. Allaoui, A. El Amrouss, A. Ourraoui, Three Solutions For A QuasiLinear Elliptic Problem, Applied Mathematics E-Notes, 13 (2013), 51-59.

[3] G. A. Afrouzi, A. Hadjian, S. Heidarkhani, Steklove problems involving the $\mathrm{p}(\mathrm{x})$-Laplacian, Electronic Journal of Differential Equations, Vol. 2014 (2014) No. 134, 1-11.

[4] S. Antontsev, S. Shmarev, Handbook of Differential Equations, Stationary Partial Differ. Equ. 3 (2006). Chap. 1

[5] B. Ricceri, A three critical points theorem revisited, Nonlinear Anal., 70 (2009), 3084-3089.

[6] G. Bonanno, A. Chinnì, Existence and multiplicity of weak solutions for elliptic Dirichlet problems with variable exponent, J. Math. Anal. Appl., 418 (2014), 812-827.

[7] G. Bonanno, S. A. Marano, On the structure of the critical set of nondifferentiable functions with a weak compactness condition, Appl. Anal., 89 (2010), 1-10.

[8] G. Bonanno, G. Molica Bisci, Infinitely many solutions for a boundary value problem with discontinuous nonlinearities, Bound. Value Probl., 2009 (2009), 1-20. 
[9] M. M. Boureanu, Infinitely many solutions for a class of degenerate anisotropic elliptic problems with variable exponent, Taiwanese Journal of Mathematics, 15 (5), 2291-2310.

[10] H. Brezis, L. Nirenberg, Remarks on finding critical points, Commun Pure Appl Math., 44 (1991), 939-963. doi:10.1002/ cpa.3160440808.

[11] J. Chabrowski, Y. Fu, Existence of solutions for $p(x)$-Laplacian problems on a bounded domain, J. Math. Anal. Appl., 306 (2005), 604-618.

[12] Y. Chen, S. Levine, R. Rao, Variable exponent, linear growth functionals in image restoration, SIAMJ. Appl. Math., 66 (2006), 1383-1406.

[13] N. T. Chung, Multiple solutions for a class of $p(x)$-Kirchhoff type problems with Neumann boundary con- ditions, Advances in Pure Appl. Math., 4 (2) (2013), 165-177.

[14] S.-G. Deng, A local mountain pass theorem and applications to a double perturbed $p(x)$-Laplacian equations, Appl. Math. Comput., 211 (2009), 234-241.

[15] S.-G. Deng, Eigenvalues of the $p(x)$-Laplacian Steklov problem, J. Math. Anal. Appl., 339 (2008), 925-937.

[16] G. Dai, Infinitely many solutions for a $p(x)$-Laplacian equation in $\mathbb{R}^{N}$, Nonlinear Anal., 71 (2009), 1133-1139.

[17] S.-G. Deng,Positive solutions for Robin problem involving the $p(x)$ Laplacian, J. Math. Anal. Appl., 360 (2009), 548-560.

[18] L. Diening, P. Hästö, A. Nekvinda, Open problems in variable exponent Lebesgue and Sobolev spaces, in: P. Drábek, J. Rákosník (Eds.), Proceedings, Milovy, Czech Republic, 2004, pp. 38-58.

[19] X. Fan, X. Han, Existence and multiplicity of solutions for $p(x)$-Laplacian equations in $\mathbb{R}^{\mathrm{N}}$, Nonlinear Anal., 59 (2004), 173-188.

[20] M. Ferrara, S. Heidarkhani, Multiple solutions for perturbed p-Laplacian boundary-value problems with impulsive effects, Electronic journal of differential equations, n. 2014 (2014), 1-14, ISSN: 1072-6691.

[21] X. Fan, D. Zhao, On the spaces $\mathrm{L}^{\mathrm{p}(\mathrm{x})}(\Omega)$ and $\mathrm{W}^{\mathrm{m}, \mathrm{p}(\mathrm{x})}(\Omega)$ J. Math. Anal. Appl., 263 (2001), 424-446. 
[22] X. Fan. Eigenvalues of the $p(x)$-Laplacian Neumann problems, Nonlinear Anal., 67:2982-2992, 2007. http://dx.doi.org/10.1016/j.na.2006.09.052.

[23] Y. Fu, X. Zhang, A multiplicity result for $p(x)$-Laplacian problem in $\mathbb{R}^{N}$, Nonlinear Anal., 70 (2009), 2261-2269.

[24] A. Ourraoui, Multiplicity results for Steklov problem with variable exponent, Applied Mathematics and Computation, 277 (2016), 34-43.

[25] A. Ourraoui, Some Results For Robin Type Problem Involving $p(x)$-Laplacian, (preprint).

[26] P. H. Rabinowitz, Minimax methods in critical point theory with applications to differential equations. In CBMS Regional Conference Series in Mathemat- ics, Vol. 65, American Mathematical Soceity, Providence (1986).

[27] M. A. Ragusa, A. Tachikawa, Regularity for minimizers for functionals of double phase with variable exponents, Adv. Nonlinear Anal., 9 (2020), $710-728$.

[28] M. Růžička, Flow of shear dependent electrorheological fluids, CR Math. Acad. Sci. Paris, 329 (1999), 393-398.

[29] M. Růžička, Electrorheological Fluids: Modeling and Mathematical Theory, Springer-Verlag, Berlin, 2002.

[30] S. Shokooh, A. Neirameh, Existence results of infinitely many weak solutions for $\mathrm{p}(\mathrm{x})$-Lapalacian-Like operators, U.P.B. Sci. Bull., Series A, Vol. 78, Iss. 4, 2016.

[31] E. Zeidler, Nonlinear Functional Analysis and its Applications, vol. II/B, Berlin, Heidelberg, New York, 1985.

[32] Q. H. Zhang, Existence and asymptotic behavior of positive solutions for variable exponent elliptic systems, Nonlinear Anal., 70 (2009), 305-316.

[33] V. Zhikov, Averaging of functionals in the calculus of variations and elasticity, Math. USSR Izv., 29 (1987), 33-66. 